\title{
The pineal and thymus gland interaction on the immune system stimulation, through the melatonin hormone effect
}

\begin{abstract}
Previous studies with magneto encephalographic (MEG) recordings in patients with various brain disorders and the use of external picoTeslaTranscranial Magnetic Stimulation (pT-TMS) showed that, stimulation of the immune system was possible, depending on the proper function of the pineal gland (PG) and the melatonin hormone effect. A more detailed study of the underlying mechanisms is proposed, especially in the case that PG is calcified and melatonin secretion is partially prevented. A specifically designed electronic device can be used for the decalcification of the PG, or alternative as a hypothesis, oral intake of low doses of melatonin hormone could be given instead of using external pT-TMS.
\end{abstract}

Objectives: The aim of this study was to investigate, that in case of PG dysfunction, external pT-TMS can be used, in order to enhance the immune system stimulation or as a hypothesis oral intake of low doses of melatonin hormone could be given against infectious agents.

Keywords: MEG, pineal, thymus, melatonin, immune system, antibodies
Volume 9 Issue 2 - 202I

\author{
Photios Anninos,' Adam Adamopoulos,' \\ Nicolia Anninou, ${ }^{2}$ Nicolaos Tsagas ${ }^{3}$ \\ 'Laboratory of Medical Physics, Department of Medicine, School \\ of Health Sciences, Democritus University of Thrace, Greece \\ ${ }^{2}$ Private Sector Veterinarian, Greece \\ ${ }^{3}$ Department of Electrical Engineering, University of Thrace, \\ Greece
}

Correspondence: Photios Anninos, Emeritus Professor, Laboratory of Medical Physics, Department of Medicine, School of Health Sciences, Democritus University of Thrace, University Campus, Alexandroupolis, Greece, Tel 6974974846,

Email pans.photios.anninos@gmail.com

Received: March 06, 2021 | Published: March 17, 2021

\section{Introduction}

The PG is shown to be an important organ of our brain which is located on the top of the third ventricle and has weight of $0.15 \mathrm{~g}$. It spends a lot of energy due to its functions. P rincipally, it secrets over the night an important hormone, the melatonin, ${ }^{1,2}$ which is involved in sleep regulation, and other important bodily activities and circadian rhythms in human subjects. ${ }^{3,4}$

The PG exerts control on several organs of the CNS through the melatonin hormone action. ${ }^{2}$ Once melatonin enters the blood, it is absorbed from the choroid plexus into the different brain centers. ${ }^{2}$ Among these centers are the following: (a) The hypothalamus, which is the central endocrine regulator. (b) The cerebellum, which is an inhibitory complicated motor machinery. Any damage on the cerebellum may leads to the development of epilepsy. ${ }^{5}$ (c) There is interaction between the cerebellum and the hippocampus, which is also controlled by the PG and plays an important role in memory functions and epilepsy. ${ }^{5}$ (d) In addition, PG is also a regulator of our immune system through the TG function ${ }^{6,7}$ which is generating the infection fighting $\mathrm{T}$ cells that neutralize foreign invaders such as virus and bacteria. ${ }^{6,7}$ These cells also help to protect the human body against infections, by the production of the five immunoglobulins $\mathrm{s}^{6,7}$ or antibodies. These antibodies are glycoproteins molecules that make up an important part of our immune system, for fighting off foreign organisms. (e) The above mentioned antibodies are classified into the following five categories: $\lg \mathrm{A}, \lg \mathrm{D}, \lg \mathrm{E}, \lg \mathrm{G}$ and $\lg \mathrm{M}$. Among them only the $\lg \mathrm{A}, \lg \mathrm{g}$ and $\lg \mathrm{M}$ have significant amounts in the human body, but all are very important contributors to humans immunity. ${ }^{6,7}$

More specifically, $\lg$ A accounts to be about $15 \%$ of the antibodies in the human system and in $6 \%$ in the blood serum. ${ }^{6}$ It has a half-life of 5 days and a total of four sites to bind antigens, which are also called epitope-binding sites and, as such, are invaders to trigger an immune reaction. ${ }^{6,7}$ The second, $\lg \mathrm{D}$, accounts for $0.2 \%$ of the serum antibodies and has two epitope-binding sites. It is found to a lesser extent in blood and lymphatic fluid. ${ }^{6,7}$ The third, $\lg$, accounts for $0.002 \%$ of serum antibody and as it is published ${ }^{6}$ plays a vital role in the immune system having two antigenic binding sites and a short half-life of two days. It is a mediator of allergic reactions and also, it takes part in chemical degradation. ${ }^{6,7}$ The fourth, $\lg$, is the dominant antibody ${ }^{6}$ in the human body accounting for the $85 \%$ of all antibodies, has a half-life of 7-23 days, the unique ability to cross placenta in pregnant women protecting the unborn fetus and newborn baby ${ }^{6}$ and the competence to neutralize viruses and killing bacteria. ${ }^{6}$ For these reasons, this antibody is prevalent in the system. ${ }^{6,7}$ The fifth, $\operatorname{lgM}$, is the most efficient of all antibodies. It has a short half-life of five days and is accounting approximately for $13-15 \%$ of the serum antibodies. As it was published ${ }^{6}$ it has 10 epitope-binding sites making it fierce adversary. ${ }^{6,7}$

As part of our previous studies with magneto encephalographic (MEG) recordings for the beneficial effect of the application of external pT-TMS on several Central Nervous System (CNS) diseases, ${ }^{6,8-13}$ the crucial role of the proper function of PG on the immunity was revealed and the importance of not to be calcified. ${ }^{6}$ In case where the PG was impaired i.e calcified (Figure 5) the melatonin secretion was partially prevented. The assessment of the PG calcification status was provided by using computing tomography (CT) in each patient with abnormal MEG activity, ${ }^{6,14}$ as it was seen by using Fast Fourier statistical analysis (FFT).

\section{Methods}

Through the application of a software program developed in our laboratory we were able to detect the amplitude of the primary dominant frequency in the power spectrum of the MEG obtained from every patient. ${ }^{8,10}$ By using FFT we could obtain maps for those frequencies from the power spectra on the MEG data from each patient. Different colors on the maps represent different dominant frequencies. The numbers in the maps squares represent the $122 \mathrm{MEG}$ channels in every part of the brain area. The colors of the dominant frequencies are red $=2 \mathrm{~Hz}$, orange $=3 \mathrm{~Hz}$, yellow $=4 \mathrm{~Hz}$, green $=5 \mathrm{~Hz}$, blue $>$ or $=6 \mathrm{~Hz}$ ) (Figure $1 \&$ Figure 2). The same procedure was also 
applied after the use of external pT-TMS (Figure $3 \&$ Figure 4). In this way we can see the effect before and after the use of pT-TMS in every patient with calcified (Figure 5) $\mathrm{PG}^{14}$ and thus impaired melatonin secretion (see figures 1-4) as an increase of the $\theta$ and $\delta$ (pathological) frequencies towards the alpha (normal) frequencies, as what we have already published. ${ }^{8,10}$

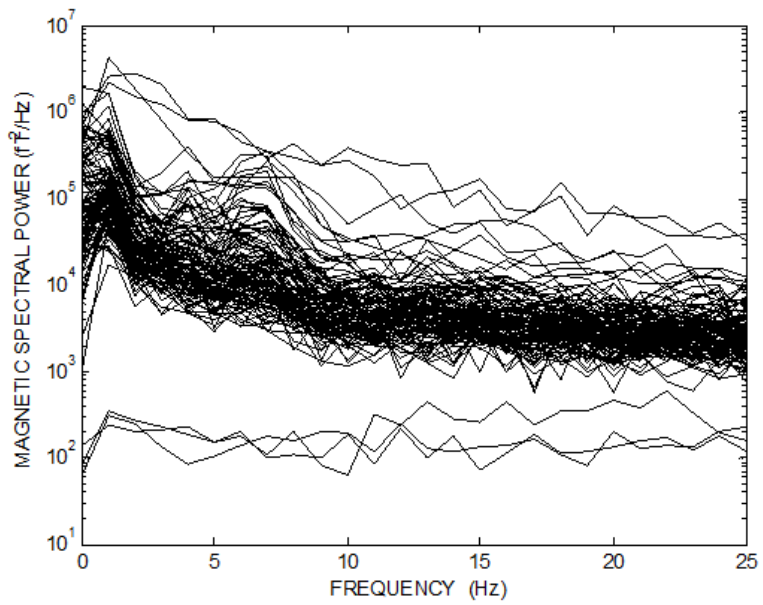

Figure I Power spectra of the I 22 channel MEG.

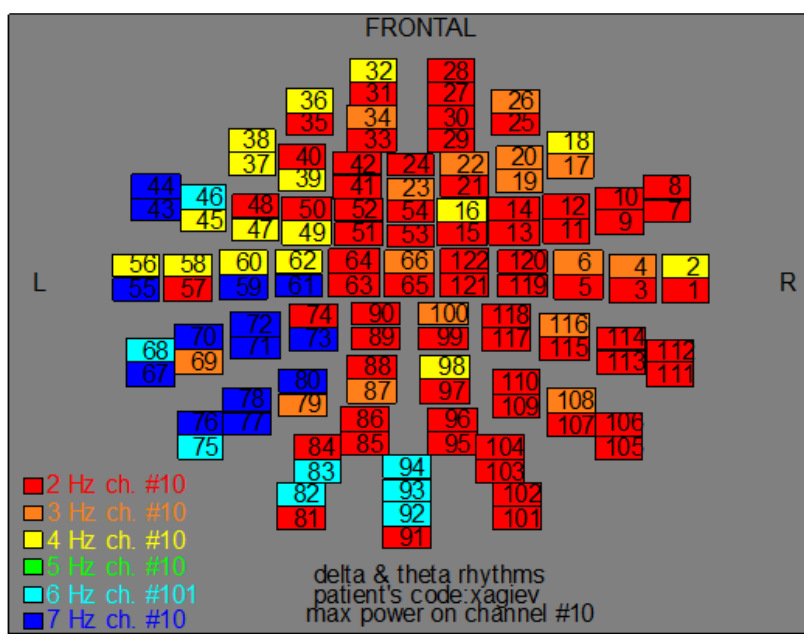

Figure 2 Brain mapping of dominant frequency for each one of the 122 MEG channels.

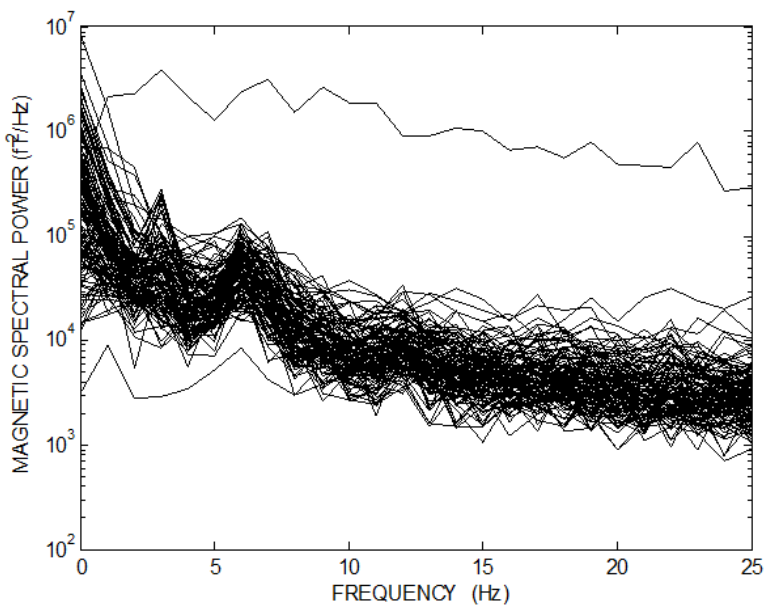

Figure 3 Power spectra of the 122 channel MEG after the application of external PT-TMS.

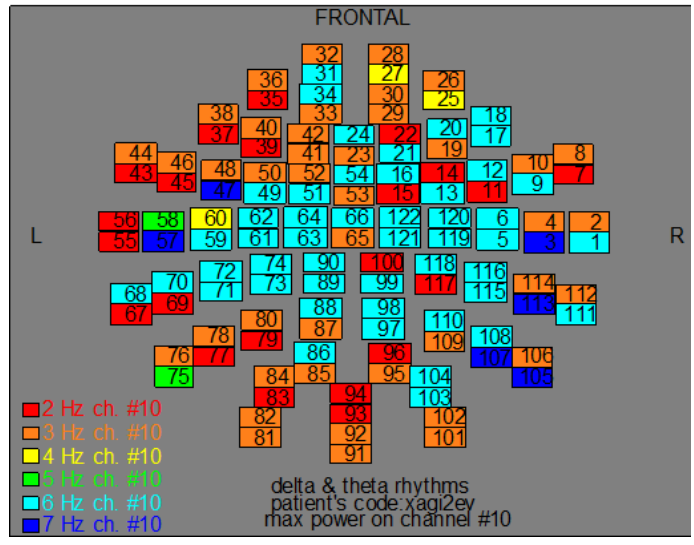

Figure 4 Brain mapping of dominant frequency for each one of the I22 MEG channels after the application of external PT-TMS.

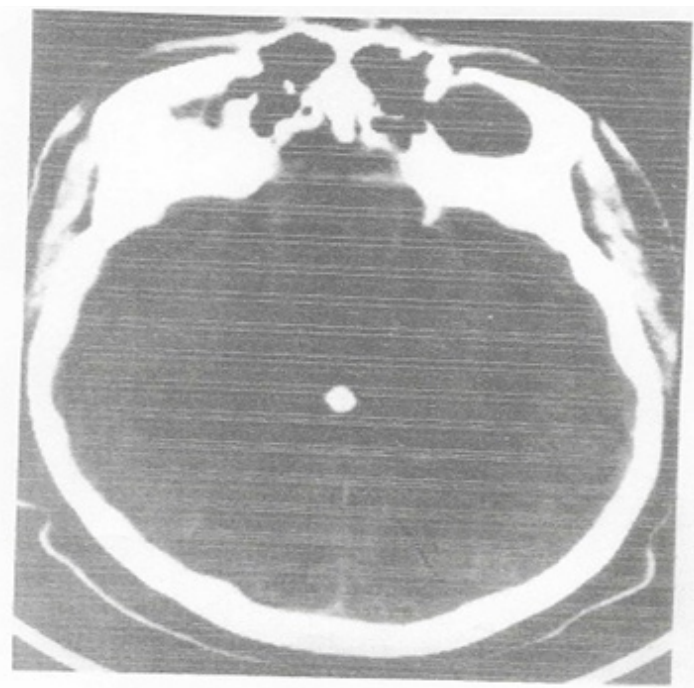

Figure 5 CT of calcified PG.

\section{Discussion}

As it is published ${ }^{6,15}$ the $P G$ has an important immunomodulatory role and function through the physiological synthesis and secretion of the melatonin hormone. These results, which we have seen by the melatonin action on different brain centers, were provided because the PG was not calcified. Once this neuroendocrine gland is affected through calcification procedures, that instantly reflect in the decreased melatonin secretion and activity. ${ }^{16}$ Therefore, so as to overcome the problem of calcification an electronic device ${ }^{17}$ was developed. This was done in order to help the PG release the melatonin hormone at normal levels..$^{6,8-13}$ By this way we were able to influence the TG to produce the proper antibodies to destroy any foreign organisms invading in the human organism. ${ }^{6,15}$

However, in order to avoid the patients to get and use the electronic device for decalcification of the PG for the normal production of melatonin hormone, as an hypothesis we could administer melatonin orally, by pills that are now widely available in drugstores, in very low doses. Oral intake of very low doses $(0.1$ to $0.3 \mathrm{mg})$ in the daytime could probably result in peak serum concentrations that are within the normal night time range. ${ }^{15,18,19}$ In this way, the TG could be influenced to produce the proper antibodies. ${ }^{6,15}$ Of course further experiments are need to be done in order to clarify our hypothesis. 


\section{Conclusion}

In conclusion, depending on the PG calcification status we have, instead of applying external pT-TMS we hypothesize that, oral intake of low doses of melatonin hormone, could possibly influence human immune system responses over infectious pathogens.

\section{Notice}

In this research assumption studies we have not included human patients due to lockdown in our country from the COVID-19 pandemic.

\section{Author's contribution}

All the authors have accepted responsibility for the entire content of this manuscript and approved submission.

\section{Funding}

This research was funded by a partnership between GGET (General Secretariat of Research and Techology, GR) and ERGO AEBE, INC, GR(Grant Number:80623).

\section{Acknowledgments}

The authors of this work are very indebted and encouraged by the comments by the Erner. Professor George Mpougioukas, Democritus University of Thrace, Alexandroupolis, Greece, and Professor Maria Lampropoulou Democritus University of Thrace, Alexandroupolis, Greece, for this scientific investigation to submit it for possible publication to the Journal of Pharmacy \& Pharmacology.

\section{Conflicts of interest}

The authors declare no conflict of interest.

\section{References}

1. Arendit J. Melatonin and the pineal gland: Influence on mammalian seasonal and circadian physiology. Rev Reprod. 1998;3(1):13-22.

2. Stankob B, Fraschini F, Reiter RJ. Melatonin binding sites in the central nervous system. Brain Res Brain Res Rev. 1991;16(3):245-256.

3. Lieberman HR, Waldhauser F, Garfield G, et al. Effects of melatonin on human mood and performance. Brain Res. 1984;323(2):201-207.

4. Vollrath L, Semm P, Gammel G. Sleep induction by intranasal application of melatonin. Adv Biosci. 1981;29(32):327-329.

5. Penfield W, Jasper H. Epilepsy and the functional anatomy of the human brain. London: Churchill; 1964. 164 p.
6. Anninos P, Adamopoulos A, Anninou N, et al. The proper function of pineal and thymus glands to control foreign organisms acting on human brain subjects. EC Neurology. 2020;12(6):59-63.

7. Csaba G. The role of brain-Pineal-Thymus in the Determination of Lifespan: The Autoimmune Aging Theory. Advances in Neuroimmune Biology. 2017;6(3-4):139-148.

8. Anninos P, Chatzimichael A, Anninou N, et al. The Effect of pT-TMS on Beta Rhythm in Children with Autism Disorder: A MEG Study. Maedica. 2019:14(4):332-342.

9. Anninos P, Kotini A, Adamopoulos A, et al. MEG recordings of patients with cerebral palsy before and after the application of pico-Tesla weak magnetic fields. J Integr Neurosci. 2019;18(1):17-21.

10. Anninos P, Kotini A, Adamopoulos A, et al. Frequency Analysis from the Effect of Pico Tesla-Transcranial Magnetic Stimulation in Migraine Patients Using Magnetoencephalography. EC Neurology. 2018;10(11):1029-1036.

11. Anninos P, Adamopoulos A, Kotini A, et al. MEG Study of Pico-Tesla Trancranial Magnetic Simulation on Patients with Depression. EC Neurology. 2017;5(3):115-122.

12. Anninos P, Adamopoulos A, Kotini A, et al. MEG evaluation of pico-Tesla external TMS on multiple sclerosis patients. Mult Scler Relat Disord. 2016;8:45-53.

13. Anninos P, Adamopoulos A, Kotini A, et al. Combined MEG and pT-TMS study in Parkinson's disease. J Integr Neurosci. 2016;15(2):145-162.

14. Sandyk R, Tsagas N, Anninos P. A Melatonin as a procovulsive hormone in humans. Int J Neurosci. 1992;63(1-2):125-135.

15. Anninos P, Anninou N, Adamopoulos A, et al. Oral Intake of Melatonin Hormone could Influence the Thymus Gland for Producing the Proper Antibodies. EC Neurology. 2020;12(8):175-176.

16. Pazo JH. Effects of melatonin on spontaneous and evoked neuronal activity in the mesencephalic reticular formation. Brain Research Bulletin. 1979;4(6):725-730.

17. Anninos P, Tsagas N. Electronic apparatus for treating epileptic individuals. USA patent 5453072A; 1995.

18. Zhdanova IV, Wurtman RJ, Morabito C, et al. Effects of Low Oral Doses of Melatonin, Given 2-4 Hours Before Habitual Bedtime, On Sleep in Normal Young Humans. American Sleep Disorders Association and Sleep Research Society. 1996;19(5):423-431.

19. Dollins AB, Zhdanova IV, Wantman RJ, et al. Effect of inducing nocturmal serum melatonin. Concentrations in daytime on sleep, mood, body temperature and performance. Proc Natl Acad Sci USA. 1994;91(5):1824-1828. 